Diabetologe $2021 \cdot 17: 703-704$

https://doi.org/10.1007/s11428-021-00812-1

Angenommen: 18. August 2021

(c) Springer Medizin Verlag $\mathrm{GmbH}$, ein Teil von Springer Nature 2021

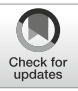

\title{
Diabetes und Psyche
}

\section{Psychiatrie ist keine "Geisteswissenschaft"}

\author{
Dieter F. Braus \\ Vitos Rheingau, Eltville, Deutschland
}

Psychische Störungen sind mit einer Lebenszeitprävalenz von bis zu $40 \%$ häufig, sie gehen mit einer erheblichen Einschränkung der Erlebnis- und Gestaltungsfähigkeit der Betroffenen einher und stellen für alle medizinischen Disziplinen, gerade auch für die Diabetologie, einen relevanten Komorbiditätsfaktor dar. Psychisch erkrankte Menschen sterben laut internationalen Befunden durchschnittlich 10 Jahre früher als die Allgemeinbevölkerung, was weitestgehend auf körperliche Erkrankungen zurückzuführen ist [1, 2]. Gleichzeitig sind psychische Störungen mit einer hohen Stigmatisierung behaftet, die sich besonders im ersten Behandlungsjahr auch ungünstig auf den Behandlungserfolg auswirkt [3]. Diese negative Haltung gegenüber Psychiatrie und psychiatrischer Behandlung beschränkt sich nicht nur auf die Betroffenen, sondern spiegelt sich in gleicher Weise auch bei Ärzt:innen und Psycholog:innen wider [4].

Es gibt enge pathophysiologische Überlappungen zwischen psychischen Störungen und der Diabetologie, wie in den Artikeln in diesem Heft aufgezeigt, außerdem können sich Psychopharmaka ungünstig auf metabolische Parameter auswirken, das Fallbeispiel von Davis-Glurich et al. ist dabei sehr eindrücklich. Auch Themen wie Motivation zur Lebensstilveränderung oder Stressreduktion durch Achtsamkeit sind praxisrelevante Aspekte der Diabetologie.

Die somatische Komorbidität und die Mortalität bei psychisch Erkrankten nehmen durch additive Gesundheitsrisiken weiter zu, etwa sozioökonomische Probleme (z. B. Arbeitslosigkeit) und Lebensstilfaktoren (z. B. körperliche Inaktivität) oder psychisches Trauma. Gleichzeitig nutzen Menschen mit chronischen psychischen
Erkrankungen die Gesundheitsleistungen nur eingeschränkt und zudem mit schlechterer Adhärenz. Auch hindern fehlende Kenntnisse zu oder Interesse an psychischen Störungen Versorger:innen daran, konstruktiv mit der somatischen und gleichzeitig psychischen Erkrankung umzugehen, wie das Fallbeispiel von Schultze et al. zeigt.

Weitere Faktoren im Zusammenhang mit der Kommunikation zwischen Ärzt:in und Patient:in können ebenfalls relevant sein, z. B. die Fragen, welche Werkzeuge zur Motivation zur Verfügung stehen oder wie Stressmanagement und Psychoeduktion optimiert werden können. Hier zeigen Leutelt et al. Möglichkeiten auf.

Darüber hinaus können psychotrope Medikamente Nebenwirkungen hervorrufen, wie Schullerus et al. beschreiben, und die Entwicklung somatischer Komorbiditäten fördern, obwohl diese Arzneimittel die Sterblichkeit bei schweren psychischen Störungen insgesamt deutlich senken.

Relevant ist für die Behandler:innen von psychischen Störungen, dass die Diagnosen in der Psychiatrie - im Gegensatz zu den meisten anderen medizinischen Disziplinen - üblicherweise auf subjektiv berichteten Symptomen und von der Ärzt:in oder psychologischen Psychotherapeuten:in beobachtbaren körpersprachlichen oder Verhaltensmustern des Betroffenen beruhen. Empathisches Zuhören, Einordnung der Subjektivität und ihrer Qualia (subjektiver Erlebnisgehalt eines mentalen Zustandes), Intuition, Selbsterfahrung des Behandelnden zur Erkennung von "Spiegel- und Schattenphänomenen", gute Beobachtungs- und Kommunikationsfähigkeit sowie Beziehungsaufbau, um nur einige der Aspekte zu benennen, spielen 
deshalb im psychiatrischen Alltag seit Jahrzehnten die zentrale Rolle [5].

Mit den Errungenschaften der neurobiologischen Forschung der letzten 3 Dekaden, insbesondere der kognitiven, affektiven und sozialen Neurowissenschaften, kam es zu einer grundlegenden Veränderung pathophysiologischer Denkmodelle [5]. Klinische Syndrome, die bisher als "psychisch" im Kontrast zu "somatisch" bzw. „organisch" betrachtet wurden, gelten in der wissenschaftlichen Welt nun als Syndrome, die auf gestörten neuronalen Netzwerken im Gehirn beruhen. Somit werden "seelische Störungen“ zu "funktionellen Hirnerkrankungen", bei denen die gleichen Zugangswege offen stehen wie bei allen anderen Erkrankungen [5]. Dies wird an den Beispielen der Angststörungen (Linsmayer et al.), Depressionen (Mahabadi et al.) oder somatoformen Störungen (Pfeiffer et al.) deutlich.

\section{॥ Gefühle und Emotionalität sind essenzielle Hirnfunktionen für einen konstruktiven Umgang mit Stress}

Gefühle, Emotionalität und Emotionsregulation sind nichts Metaphysisches, sie sind essenzielle Hirnfunktionen für einen konstruktiven Umgang mit Stress- und jeglicher Krankheitserfahrung. Fehlfunktionen sind Grundlage affektiver Störungen, aber auch anderer stressassoziierter Erkrankungen wie somatoformer Störungen, Angststörungen, Autoimmunerkrankungen, $\mathrm{Hy}$ pertonie und Diabetes mellitus Typ 2. Die Förderung von Resilienz hat dabei eine große präventive Bedeutung - für all diese Erkrankungen. Durch Berücksichtigung häufiger psychiatrischer Komorbiditäten und ganzheitliche Betrachtung des Betroffenen können der Alltag in der diabetologischen Praxis bereichert und eine noch bessere Therapiequalität erzielt werden.

\section{Korrespondenzadresse}

Prof. Dr. Dieter F. Braus

Vitos Rheingau

Kloster-Eberbach-Straße 4, 65123 Eltville,

Deutschland

dieter.braus@vitos-rheingau.de

Interessenkonflikt. D.F. Braus hat in den letzten Jahren über dessen Arbeitgeber produktneutrale
Vorträge gehalten für med-update, streamed-up, Janssen, Lilly, TAD, Takeda, Medice, Bayer, Lundbeck, Pfizer, Recordati, Sanofi, Sunovion, Amgen, BerlinChemie, MSD Sharp \& Dohme, Servier.

\section{Literatur}

1. Walker ER, McGee RE, Druss BG (2015) Mortality in mental disorders and global disease burden implications: a systematic review and metaanalysis. JAMA Psychiatry 72(4):334-341

2. Schneider F, Erhart M, Hewer W, Loeffler LA, Jacobi F (2019) Mortality and medical comorbidity in the severely mentally ill. Dtsch Arztebl Int 116(23-24):405-411. https://doi.org/10.3238/ arztebl.2019.0405

3. Oexle N, Müller M, Kawohl W, Xu Z, Viering S, Wyss C, Vetter S, Rüsch N (2018) Self-stigma as a barrier to recovery: a longitudinal study. Eur Arch Psychiatry Clin Neurosci 268(2):209-212

4. Angermeyer MC, van der Auwera S, Carta MG, Schomerus G (2017) Public attitudes towards psychiatry and psychiatric treatment at the beginning of the 21 st century: a systematic review and meta-analysis of population surveys. World Psychiatry 16(1):50-61

5. Braus DF (2014) EinBlick ins Gehirn, eine andere Einführung in die Psychiatrie. Thieme, Stuttgart

\section{Diabetes Update 2022 Das Neueste in der Diabetologie}

11. und 12. März 2022 in Mainz

Umfangreiches Update-Wissen in zwei kompakten Fortbildungstagen: all das bietet Ihnen das Diabetes Update in Mainz mit parallelem Livestream.

Das Update-Konzept: Die wichtigsten Neuerungen des vergangenen Jahres aus der Diabetologie werden unter der wissenschaftlichen Leitung von Prof. Dr. Andreas Hamann (Bad Homburg), Dr. Helmut Kleinwechter (Kiel), Prof. Dr. Stephan Martin (Düsseldorf) und Prof. Dr. Michael Stumvoll (Leipzig) kritisch selektiert, analysiert und zusammengefasst. Die Relevanz für den Klinik- und Praxisalltag der Ärztinnen und Ärzte steht dabei im Vordergrund, und der ausführlichen Diskussion mit den referierenden Expertinnen und Experten wird viel Raum gegeben.

Das aktuelle Programm enthält neben den bewährten Kerngebieten die neuen Vorträge »Hot Topic: Diabetes und Psyche« und »Hot Topic: Wundmanagement«.

Zum Gesamtpaket der Teilnahme gehören neben den umfangreichen Seminarunterlagen, der Download aller Vortragspräsentationen und die Vorträge im Nachgang als Video-on-Demand.

Weitere Informationen zu Programm, Referierenden und zur Anmeldung: www.diabetes-update.com

Veranstalter: med update $\mathrm{GmbH}$ Hagenauer Straße 53 65203 Wiesbaden 